PPM1B and P-IKK $\beta$ expression levels correlated inversely with rat gastrocnemius atrophy after denervation

Jian Wei and Bing-Sheng Liang

The Brazilian Journal of Medical and Biological Research is partially financed by

\section{贵CNPq}

da Ciência e Tecnologia

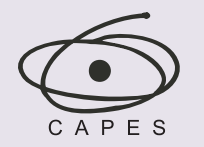

Ministério da Educação
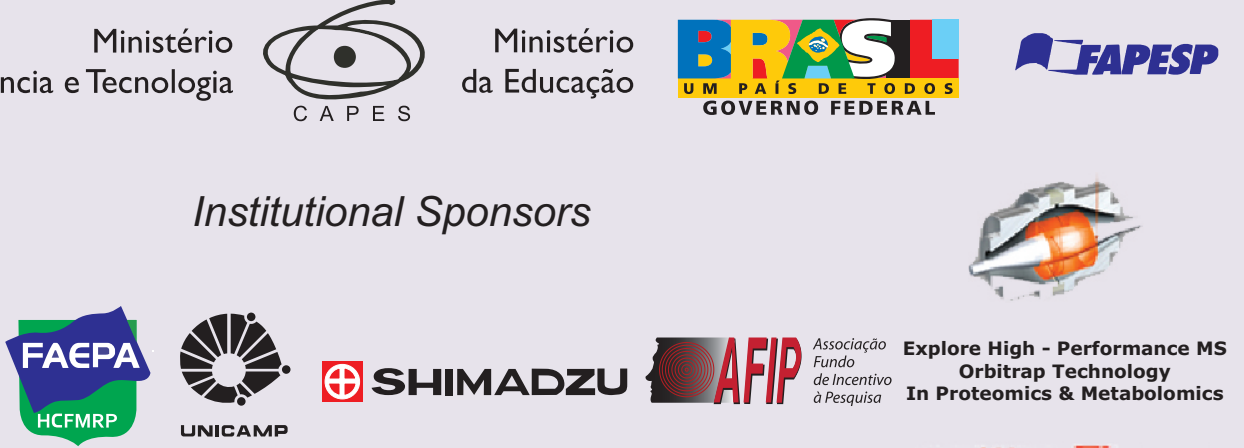

Institutional Sponsors

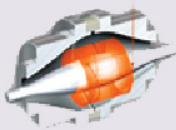

Ф SHIMADZU UNICAMP

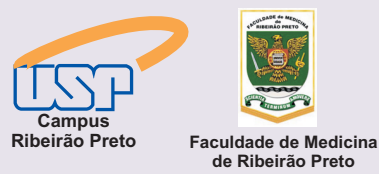

de Ribeirão Preto 


\title{
PPM1B and P-IKK $\beta$ expression levels correlated inversely with rat gastrocnemius atrophy after denervation
}

\author{
Jian Wei and Bing-Sheng Liang
}

Department of Orthopedics, the Second Hospital, Shanxi Medical University, Taiyuan, China

\begin{abstract}
Activated inhibitor of nuclear factor-kB kinase $\beta$ (IKK $\beta$ ) is necessary and sufficient for denervated skeletal muscle atrophy. Although several studies have shown that $\mathrm{Mg}^{2+} / \mathrm{Mn}^{2+}$-dependent protein phosphatase 1B (PPM1B) inactivated IKK $\beta$, few studies have investigated the role of PPM1B in denervated skeletal muscle. In this study, we aim to explore the expression and significance of PPM1B and phosphorylated IKK $\beta(P-I K K \beta)$ during atrophy of the denervated gastrocnemius. Thirty young adult female Wistar rats were subjected to right sciatic nerve transection and were sacrificed at 0 (control), 2, 7, 14, and 28 days after denervation surgery. The gastrocnemius was removed from both the denervated and the contralateral limb. The muscle wet weight ratio was calculated as the ratio of the wet weight of the denervated gastrocnemius to that of the contralateral gastrocnemius. RT-PCR and Western blot analysis showed that mRNA and protein levels of PPM1B were significantly lower than those of the control group at different times after the initiation of denervation, while P-IKK $\beta$ showed the opposite trends. PPM1B protein expression persistently decreased while P-IKK $\beta$ expression persistently increased for 28 days after denervation. PPM1B expression correlated negatively with P-IKK $\beta$ expression by the Spearman test, whereas decreasing PPM1B expression correlated positively with the muscle wet weight ratio. The expression levels of PPM1B and P-IKK $\beta$ were closely associated with atrophy in skeletal denervated muscle. These results suggest that PPM1B and P-IKK $\beta$ could be markers in skeletal muscle atrophy.
\end{abstract}

Key words: Muscular atrophy; Denervation; PPM1B; P-IKKß; IKKß; RT-PCR; Western blot

\section{Introduction}

Muscle atrophy can be caused by many genetic and environmental factors, including denervation $(1,2)$. Currently, no effective treatment exists that can reverse the progression of muscle atrophy once initiated. Many strategies that efficiently promote muscle growth cannot prevent the muscle atrophy process (3), suggesting that diverse mechanisms maintain the muscle mass in different contexts. In cases of acute muscle atrophy, active elimination of the myonuclei is considered to be important when load is lacking; in cases of chronic muscle atrophy, the impairment of muscle repair is important (3). Targeting the proper molecules during muscle atrophy is critical to the reversal or cure the progression of atrophy. An understanding of the molecular mechanisms underlying the initiation and progression of muscle atrophy may have therapeutic implications for clinical treatment or drug targets.

Many signaling pathways and molecules, including the IGF-1/PI3K/Akt pathway, muscle ring finger 1, and muscle atrophy F-box, both of which are ubiquitin ligases (4), mediate muscle atrophy caused by denervation or disuse. Previous studies have suggested that the inhibitor of the nuclear factor-kB kinase $\beta$ (IKK $\beta / N F-k B$ ) pathway may be important in the development of muscle atrophy in denervated muscles (5-7), and that the phosphorylated inhibitor of nuclear factor-KB kinase $\beta$ (P-IKK $\beta$ ) might be the critical arbiter in this process $(5,8-10)$. IKK $\beta$ phosphorylation and activation is an important step in the activation of NF-KB by tumor necrosis factor- $\alpha$ (TNF- $\alpha)$ (11). Upon stimulation by TNF- $\alpha$, interleukin-1 (IL-1), and other cytokines, IKK $\beta$ undergoes rapid phosphorylation and activation, whereas the IKB kinase (IKK) complex undergoes ubiquitination for proteasome degradation. This process exposes the nuclear localization signal area of NF-KB and leads to its nuclear transport, which in turn regulates the expression of downstream genes and results in muscle atrophy (9).

$\mathrm{Mg}^{2+} / \mathrm{Mn}^{2+}$-dependent protein phosphatase 1B (PPM1B

Correspondence: Bing-Sheng Liang, Department of Orthopedics, Shanxi Medical University Second Hospital, 382 Wuyi Road, Xinghualing District, Taiyuan 030001, China. Fax: +86-0351-758-2190. E-mail: liangbingsheng@yahoo.com.cn

Received November 1, 2011. Accepted May 3, 2012. Available online May 18, 2012. Published August 3, 2012. 
or PP2C) is a member of the Ser/Thr protein phosphatase (PP2C) family (12-15). There are 6 different splice variants of PPM1B, which show a broad specificity for different functions. PPM1B is highly expressed in heart and skeletal muscle and often negatively regulates cell reaction pathways (16-18). For instance, PPM1B can dephosphorylate cyclin-dependent kinase, thus regulating stress response, cell cycle, and actin cytoskeleton changes. PPM1B can suppress the kinase cascade effects of stress-induced p38 and c-Jun N-terminal kinases. PPM1B can also dephosphorylate and inactivate transforming growth factor- $\beta$ (TGF- $\beta$ )-activated kinase 1 and mitogen-activated protein kinase kinase kinase (MKKK) induced by stress, IL-1 stimulation, and/or TGF- $\beta$ treatment to down-regulate the activity of the NF-KB signaling pathway (12,19-24). In HeLa cells, PPM1B was shown to dephosphorylate serine 177 and 181 in P-IKK $\beta$, thereby reducing the activity of IKK $\beta$ (12). Moreover, PPM1B overexpression can block the activation of the IKK $\beta / N F-k B$ pathway induced by TNF- $\alpha$ treatment (12). However, the roles of PPM1B in denervated skeletal muscle cells have not been fully investigated.

In this study, using a rat model of denervation-induced skeletal muscle (gastrocnemius) atrophy, we examined variations in the expression levels of PPM1B and P-IKK 3 . We found that their expression levels correlated inversely with muscle atrophy after denervation. The results suggest that PPM1B and P-IKK $\beta$ could be markers in skeletal muscle atrophy.

\section{Material and Methods}

\section{Animals and surgery}

Thirty young adult female Wistar rats (250-300 g) were provided by the Experimental Animal Center of Shanxi Medical University. The animals were randomly and evenly assigned to 5 groups of 6 rats each, including a control group (0 day) and 2-, 7-, 14-, and 28-day groups.

For surgery, the rats were anesthetized with an intraperitoneal injection of $10 \%$ chloral hydrate $(2 \mathrm{~mL} / \mathrm{kg})$. After the animals were sedated, a posterior incision was made in the right lower limb for sciatic nerve transection (removal of $1.0 \mathrm{~cm}$ ). The proximal end of the nerve was turned over and sutured to the surrounding tissues to prevent reinnervation of the gastrocnemius muscle. The control group was anesthetized for sham surgery, in which the nerve was exposed but not transected. The animals were sacrificed at 0 (control), 2, 7, 14, or 28 days after denervation surgery. The bilateral gastrocnemius muscles were harvested for analysis.

This study was approved by the Ethics Committee for the Scientific Use of Animals of Shanxi Medical University.

\section{Reagents}

In addition to the materials mentioned in the text, the following reagents and kits were used: real-time PCR (RT-
PCR) kit (MBI, China), DNA and protein markers (Sigma, USA), protein extraction reagent (Bio Co., Ltd., China), and $\beta$-actin (C4) antibody (Santa Cruz, USA).

\section{RT-PCR}

The RT-PCR primers were designed with the Primer 3.0 software package and were synthesized by Shanghai Biological Engineering Co., Ltd. (China). The following primers were used: rat PPM1B, upstream = 5'-gggtcgtt agcagtgtctcgt-3', downstream = 5'-gctcctcattgctcatcac atc-3'; GADPH, upstream = 5'-tgaacgggaagctcactgg-3', downstream $=5$ '-tccaccacctgttgctgta-3'. Total mRNA was extracted with the Trizol reagent (Shanghai Sangon Technology Service, China) for reverse transcription synthesis of cDNA. The resulting cDNA was amplified by PCR with real-time monitoring of the fluorescence accumulation. The $\mathrm{Ct}$ value was set as the number of cycles needed for the required threshold of fluorescence. The $2^{-\Delta \Delta \mathrm{Ct}}$ method was used for data analysis (25).

\section{Western blot}

Rat denervated gastrocnemius muscles were dissected, frozen in liquid nitrogen, pulverized, and homogenized in $20 \mathrm{mM}$ Tris- $\mathrm{HCl}, 4 \%$ (w/v) SDS, 1 mM phenylmethylsulfonyl fluoride, and $1 \mu \mathrm{m}$ each of leupeptin and pepstatin A. Protein concentrations were determined using an SDS-compatible protein assay. Protein samples were mixed with loading buffer, subjected to SDS-PAGE (10\%), and transferred electrophoretically to Immobilon-NC membranes. Gels with identical samples were stained with Coomassie brilliant blue and used as an additional control of equilibration of protein loading. Samples were transferred to a nitrocellulose membrane, which was blocked in Blotto buffer containing $10 \%$ skim milk in Tris-buffered saline with Tween 20 (TBST) and incubated overnight at $4^{\circ} \mathrm{C}$ with a rabbit anti-mouse IKK $\beta$ or P-IKK $\alpha / \beta$ antibody (1:1000; Cell Signaling, USA) or goat anti-mouse PPM1B polyclonal antibody (1:1000; Santa Cruz). After washing the membrane 4 times with TBST for 15 min each, the membrane was incubated with horseradish peroxidase-labeled goat anti-rabbit IgG secondary antibody (1:1000; Beijing Zhongshan Golden Bridge Biotechnology Co., Ltd., China) at room temperature for $2 \mathrm{~h}$, followed by another set of four 15-min washes with TBST. The membrane was processed for enhanced chemiluminescence using appropriate reagents (Santa Cruz) and was imaged by gray-scale scanning using a GS-800 Densitometer (Bio-Rad, USA).

\section{Wet weight of the gastrocnemius muscle}

The bilateral gastrocnemius muscles were harvested entirely from the starting point of the femoral condyle to the end point of the calcaneal tubercle and were measured immediately by an accurate electronic balance (1/10,000, Mettler Toledo AL104, Switzerland). The ratio between the wet weights of the denervated 
and contralateral muscles was calculated.

\section{Statistical analysis}

SPSS 13.0 was used for statistical analyses, and the results are reported as the means \pm SD. The ImageJ software was used for Western blot image analysis. Analysis of variance (ANOVA) was used for comparison of many groups. The Dunnett method was used to compare two groups, and the Spearman method was used for correlation analysis. Differences with a $P$ value $<0.05$ were considered to be statistically significant.

\section{Results}

\section{Denervated rat gastrocnemius muscle shows temporal changes of PPM1B mRNA expression}

Quantitative analysis revealed a significant and consistent decrease in the PPM1B mRNA turnover measured by RT-PCR during the denervation phase of skeletal muscle, which started as early as 2 days after denervation surgery and lasted at least 28 days. The PPM1B mRNA expression decreased to $41 \%$ of that observed in the control group at 2 days, and to $7 \%$ of control at 28 days. The mRNA expression levels were significantly different from those of the control group at all times tested (Table 1).

The PPM1B, P-IKK $\beta$, and IKK $\beta$ protein levels show time-dependent changes in the rat denervated gastrocnemius

The Western blot results for the PPM1B protein revealed trends that were similar to but smaller than the mRNA results. The PPM1B protein level began to decrease at 2 days after denervation $(P>0.05)$. Significant changes compared to the control group were evident from 7 days through 28 days after denervation. The PPM1B protein level in the denervated groups decreased from $56 \%$ of that in the control group at 7 days to $24 \%$ of that in the control group at 28 days (Figure 1, Table 1).

In contrast, the $\mathrm{P}-\mathrm{IKK} \beta$ protein days (Dunnett test). nett test). levels began to increase soon after denervation, reaching levels $137 \%$ of those in the control group at 2 days. The increase lasted for at least 28 days, when the P-IKK $\beta$ level of the denervated group was $174 \%$ of that in the control group. The P-IKK $\beta$ protein levels were significantly different from control at all times studied (Figure 1, Table 1). The IKK $\beta$ protein expression consistently decreased with time after denervation $(\mathrm{P}<0.05$ at all times compared to control); however, the magnitude of the changes was smaller than that observed for PPM1B or P-IKK $\beta$ (Figure 1, Table 1).

\section{The wet weight ratio of the gastrocnemius muscle varies with time after denervation}

The muscle wet weight ratio was calculated as the ratio of the wet weight of the injured side to that of the contralateral muscles. After denervation, the muscle wet weight

Table 1. Effects of muscle denervation on PPM1B mRNA turnover and protein levels of PPM1B, P-IKK $\beta$, and IKK $\beta$ and the muscle wet weight ratio.

\begin{tabular}{|c|c|c|c|c|c|}
\hline & \multicolumn{5}{|c|}{ Days after denervation } \\
\hline & 0 & 2 & 7 & 14 & 28 \\
\hline PPM1B mRNA & 1.00 & $0.41 \pm 0.08^{*}$ & $0.32 \pm 0.03^{*}$ & $0.14 \pm 0.04^{*}$ & $0.07 \pm 0.02^{*}$ \\
\hline PPM1B & $1.57 \pm 0.07$ & $1.55 \pm 0.05$ & $0.88 \pm 0.04^{*}$ & $0.59 \pm 0.06^{*}$ & $0.38 \pm 0.04^{*}$ \\
\hline P-IKK $\beta$ & $0.73 \pm 0.04$ & $1.00 \pm 0.05^{*}$ & $1.14 \pm 0.07^{*}$ & $1.26 \pm 0.04^{*}$ & $1.27 \pm 0.06^{*}$ \\
\hline $\mathrm{IKK} \beta$ & $1.58 \pm 0.08$ & $1.43 \pm 0.05^{\star}$ & $1.33 \pm 0.05^{*}$ & $1.31 \pm 0.07^{\star}$ & $1.18 \pm 0.06^{*}$ \\
\hline Wet weight ratio & $1.01 \pm 0.02$ & $0.99 \pm 0.03$ & $0.85 \pm 0.04^{*}$ & $0.52 \pm 0.08^{*}$ & $0.33 \pm 0.05^{*}$ \\
\hline
\end{tabular}

PPM1B $=\mathrm{Mg}^{2+} / \mathrm{Mn}^{2+}$-dependent protein phosphatase 1B; IKK $\beta=$ inhibitor of nuclear factor$\mathrm{KB}$ kinase $\beta ; \mathrm{P}$-IKK $\beta=$ phosphorylated IKK $\beta$. ${ }^{*} \mathrm{P}<0.05$ compared to the control group at 0
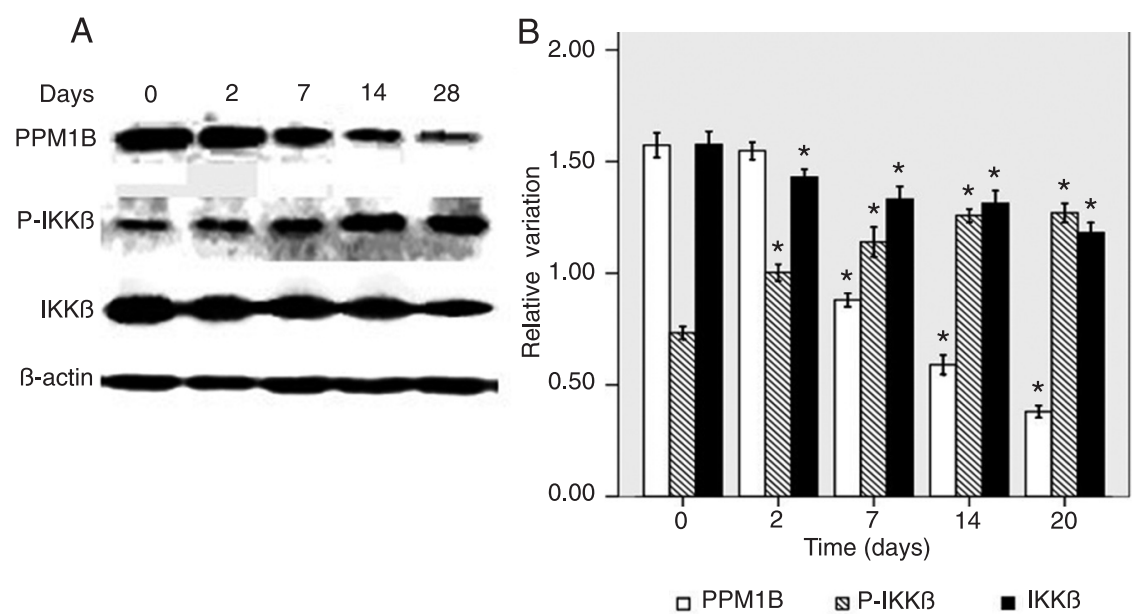

Figure 1. $A$, Western blot showing the effect of denervation of rat gastrocnemius muscle on protein levels of PPM1B, P-IKK 3 , and IKK $3 . B$, Gray-scale analysis of staining of PPM1B, $\mathrm{P}-\mathrm{IKK} \beta$, and IKK $\beta$ proteins in Western blot. Data are reported as means \pm SD. PPM1B $=$ $\mathrm{Mg}^{2+} / \mathrm{Mn}^{2+}$-dependent protein phosphatase $1 \mathrm{~B}$; IKK $\beta=$ inhibitor of nuclear factor-KB kinase $\beta ; \mathrm{P}$-IKK $\beta=$ phosphorylated IKK $\beta$. ${ }^{*} \mathrm{P}<0.05$ compared to the control group at 0 days (Dun- 
ratio decreased with time. The differences compared to control were significant starting at 7 days after denervation. At 7,14 , and 28 days, the muscle wet weight ratios of the denervated groups were 85,51 , and $33 \%$ of those in the control group, respectively (Table 1).

\section{Changes in the PPM1B and P-IKK $\beta$ expression levels correlated with the muscle wet weight ratio}

The muscle wet weight ratio is a reliable indicator of muscle atrophy, with lower ratio values indicating more severe atrophy. We performed a correlation analysis by the Spearman method between changes in the muscle wet weight ratio and changes in the mRNA turnover rates of PPM1B and P-IKK $\beta$. Changes in the PPM1B mRNA and protein expression levels correlated positively with changes in the muscle wet weight ratio $(r=0.950, P<0.01$ for mRNA; $r=0.897, P<0.01$ for protein). Changes in the level of the $\mathrm{P}$-IKK $\beta$ protein correlated negatively $(r=-0.884, \mathrm{P}<0.01)$ with changes in the muscle wet weight ratio after muscle denervation. Changes in the protein expression levels of PPM1B and P-IKK $\beta$ correlated negatively $(r=-0.878, P<$ 0.01; Figure 2).

\section{Discussion}

We used a rat model of atrophy of the denervated gastrocnemius muscle to investigate underlying molecular changes in the IKK $\beta / N F-K B$ pathway. The mRNA- and/or protein-level changes of PPM1B, IKK $\beta$, and P-IKK $\beta$ were analyzed by RT-PCR and/or Western blot at different times after denervation.

The protein expression levels of IKK $\beta$ showed a sustained decrease from 2 to 28 days after denervation. The $\mathrm{P}$-IKK $\beta$ protein levels showed a sustained increase over the same period of time and correlated negatively with changes in the muscle wet weight ratio. The contrasting changes of IKK and P-IKK $\beta$ after muscle denervation confirmed that the IKK $\beta$ phosphorylation step in the IKKß/ NF-KB pathway is important in determining the progression of muscle atrophy. Moreover, the ratio between IKK $\beta$ and $\mathrm{P}$-IKK $\beta$ could be considered a criterion for evaluating the progress of muscle atrophy.

Similarly, the PPM1B mRNA and protein expression levels in the gastrocnemius muscle displayed a sustained decrease that began 2 days after denervation surgery and lasted the entire 28-day observation period. The changes in PPM1B expression were positively and linearly correlated with the changes in the muscle wet weight ratio. Taken together, these results suggest that the altered expression levels of P-IKK $\beta$ and PPM1B are closely associated with the development of skeletal muscle atrophy after denervation.

Prajapati et al. (26) have reported that PPM1B negatively

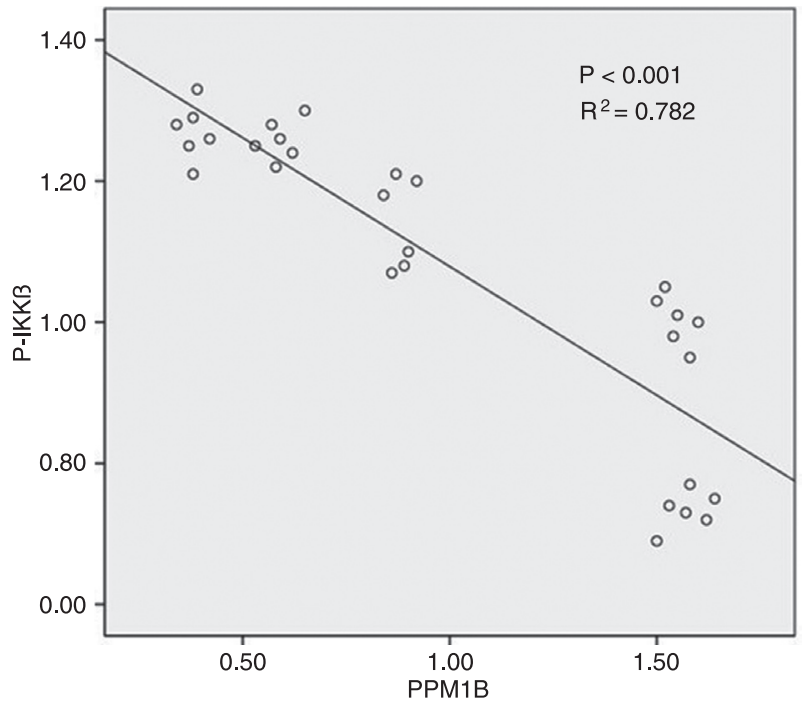

Figure 2. Correlation analysis of protein expression levels of $\mathrm{Mg}^{2+} / \mathrm{Mn}^{2+}$-dependent protein phosphatase 1B (PPM1B) and phosphorylated inhibitor of nuclear factor-KB kinase (P-IKK $\beta)$.

regulates IKK $\beta$ kinase activity. Sun et al. (12) reported that TNF- $\alpha$ induces IKK $\beta$ phosphorylation and activation. PPM1B bound to P-IKK $\beta$, subsequently dephosphorylating P-IKK at Ser177 and Ser181. This process reduced the activity of IKK $\beta$ and terminated IKK $\beta$-mediated NF-KB activation. Our current results demonstrate that PPM1B expression correlated negatively with $\mathrm{P}$-IKK $\beta$ protein expression. As skeletal muscle atrophy increased, PPM1B expression gradually decreased, while P-IKK $\beta$ expression gradually increased. However, we cannot conclude that the correlations indicate a cause/effect relationship, which mediates the pathogenesis of denervated atrophy. Further identification of PPM1B function will help elucidate the molecular mechanism of muscle atrophy after denervation.

The present study demonstrates some primary changes in PPM1B and the IKK $\beta / N F-K B$ pathway. After denervation, expression of PPM1B in skeletal muscle was downregulated, expression of P-IKK $\beta$ was up-regulated, and their expression levels correlated negatively with muscle atrophy. Thus, PPM1B might be involved in denervationinduced muscle atrophy. These results provide potential targets for molecular and genetic therapies or drug targets for pharmacological approaches to treat muscle atrophy.

\section{Acknowledgments}

Bing-Sheng Liang designed the experiments and provided the funding; Jian Wei was responsible for the experiments, data analysis and the manuscript preparation. 


\section{References}

1. Vinciguerra M, Musaro $A$, Rosenthal $N$. Regulation of muscle atrophy in aging and disease. Adv Exp Med Biol 2010; 694: 211-233.

2. Murton AJ, Greenhaff PL. Muscle atrophy in immobilization and senescence in humans. Curr Opin Neurol 2009; 22: 500-505.

3. Barton E, Morris $\mathrm{C}$. Mechanisms and strategies to counter muscle atrophy. J Gerontol A Biol Sci Med Sci 2003; 58: M923-M926.

4. Zhang P, Chen X, Fan M. Signaling mechanisms involved in disuse muscle atrophy. Med Hypotheses 2007; 69: 310321.

5. Li H, Malhotra S, Kumar A. Nuclear factor-kappa B signaling in skeletal muscle atrophy. $\mathrm{J} \mathrm{Mol} \mathrm{Med} \mathrm{2008;} \mathrm{86:} \mathrm{1113-}$ 1126.

6. Sandri M. Signaling in muscle atrophy and hypertrophy. Physiology 2008; 23: 160-170.

7. Kandarian SC, Jackman RW. Intracellular signaling during skeletal muscle atrophy. Muscle Nerve 2006; 33: 155-165.

8. Acharyya S, Villalta SA, Bakkar N, Bupha-Intr T, Janssen PM, Carathers M, et al. Interplay of IKK/NF-kappaB signaling in macrophages and myofibers promotes muscle degeneration in Duchenne muscular dystrophy. J Clin Invest 2007; 117: 889-901.

9. Cai D, Frantz JD, Tawa NE Jr, Melendez PA, Oh BC, Lidov HG, et al. IKKbeta/NF-kappaB activation causes severe muscle wasting in mice. Cell 2004; 119: 285-298.

10. Mourkioti F, Kratsios P, Luedde T, Song YH, Delafontaine P, Adami R, et al. Targeted ablation of IKK2 improves skeletal muscle strength, maintains mass, and promotes regeneration. J Clin Invest 2006; 116: 2945-2954.

11. Karin M, Ben-Neriah Y. Phosphorylation meets ubiquitination: the control of NF-[kappa]B activity. Annu Rev Immunol 2000; 18: 621-663.

12. Sun W, Yu Y, Dotti G, Shen T, Tan X, Savoldo B, et al. PPM1A and PPM1B act as IKKbeta phosphatases to terminate TNFalpha-induced IKKbeta-NF-kappaB activation. Cell Signal 2009; 21: 95-102.

13. Wang $Y$, Dow EC, Liang YY, Ramakrishnan R, Liu H, Sung TL, et al. Phosphatase PPM1A regulates phosphorylation of Thr-186 in the Cdk9 T-loop. J Biol Chem 2008; 283: 3357833584.

14. Watanabe $\mathrm{T}$, Ohnishi M, Kobayashi T, Oishi M, Tamura S. The mouse protein phosphatase 2Cbeta (Ppm1b) gene maps to the distal part of mouse chromosome 17. Genomics 1996; 33: 147-149.

15. Wang Y, Santini F, Qin K, Huang CY. A Mg $\left({ }^{2+}\right)$-dependent, $\mathrm{Ca}\left({ }^{2+}\right)$-inhibitable serine/threonine protein phosphatase from bovine brain. J Biol Chem 1995; 270: 25607-25612.

16. Sande JB, Sjaastad I, Hoen IB, Bokenes J, Tonnessen T, Holt E, et al. Reduced level of serine(16) phosphorylated phospholamban in the failing rat myocardium: a major contributor to reduced SERCA2 activity. Cardiovasc Res 2002; 53: 382-391.

17. Travis SM, Welsh MJ. PP2C gamma: a human protein phosphatase with a unique acidic domain. FEBS Lett 1997; 412: 415-419.

18. Terasawa T, Kobayashi T, Murakami T, Ohnishi M, Kato $\mathrm{S}$, Tanaka $\mathrm{O}$, et al. Molecular cloning of a novel isotype of $\mathrm{Mg}\left({ }^{2+}\right)$-dependent protein phosphatase beta (type $2 \mathrm{C}$ beta) enriched in brain and heart. Arch Biochem Biophys 1993; 307: 342-349.

19. Sasaki M, Ohnishi M, Tashiro F, Niwa H, Suzuki A, Miyazaki $\mathrm{J}$, et al. Disruption of the mouse protein Ser/Thr phosphatase 2Cbeta gene leads to early pre-implantation lethality. Mech Dev 2007; 124: 489-499.

20. Lu J, Wu DM, Zheng YL, Hu B, Zhang ZF, Shan Q, et al. Quercetin activates AMP-activated protein kinase by reducing $\mathrm{PP} 2 \mathrm{C}$ expression protecting old mouse brain against high cholesterol-induced neurotoxicity. J Pathol 2010; 222: 199-212.

21. Henmi T, Amano K, Nagaura $\mathrm{Y}$, Matsumoto K, Echigo S, Tamura S, et al. A mechanism for the suppression of interleukin-1-induced nuclear factor kappaB activation by protein phosphatase 2Ceta-2. Biochem J 2009; 423: 71-78.

22. Brody MS, Stewart V, Price CW. Bypass suppression analysis maps the signalling pathway within a multidomain protein: the RsbP energy stress phosphatase $2 \mathrm{C}$ from Bacillus subtilis. Mol Microbiol 2009; 72: 1221-1234.

23. Krieglstein J, Hufnagel B, Dworak M, Schwarz S, Kewitz $\mathrm{T}$, Reinbold M, et al. Influence of various fatty acids on the activity of protein phosphatase type $2 \mathrm{C}$ and apoptosis of endothelial cells and macrophages. Eur J Pharm Sci 2008; 35: 397-403.

24. Flajolet M, Rakhilin S, Wang H, Starkova N, Nuangchamnong $\mathrm{N}$, Nairn $\mathrm{AC}$, et al. Protein phosphatase $2 \mathrm{C}$ binds selectively to and dephosphorylates metabotropic glutamate receptor 3. Proc Natl Acad Sci U S A 2003; 100: 1600616011.

25. Livak KJ, Schmittgen TD. Analysis of relative gene expression data using real-time quantitative PCR and the 2(-Delta Delta C(T)) Method. Methods 2001; 25: 402-408.

26. Prajapati S, Verma U, Yamamoto Y, Kwak YT, Gaynor RB. Protein phosphatase 2 Cbeta association with the lkappaB kinase complex is involved in regulating NF-kappaB activity. J Biol Chem 2004; 279: 1739-1746. 